\title{
EVALUAR LA DOCENCIA EN LÍNEA: RETOS Y COMPLEJIDADES
}

\author{
(EVALUATING ONLINE TEACHING: CHALLENGES AND COMPLEXITIES)
}

\author{
Benilde García Cabrero \\ Vania Jocelyn Pineda Ortega \\ Universidad nacional Autónoma de México, UNAM (México).
}

\section{RESUMEN}

Se describen una serie de consideraciones en torno al rol del docente en escenarios virtuales de enseñanza-aprendizaje relacionados con los modelos psicopedagógicos que han dominado la escena de la educación a distancia desde sus inicios hasta nuestros días.

Se argumenta que tanto el tipo de modelo psicopedagógico, como los dispositivos tecnológicos, posibilitan o restringen las dimensiones que configuran la práctica docente en las modalidades de educación a distancia. Estos dos factores (posibilidades y restricciones), deben tomarse en cuenta en el diseño de la evaluación de la docencia en entornos virtuales.

Palabras clave: evaluación de la docencia en línea, instrucción asistida por computadora, roles docentes en entornos virtuales, educación en línea, tutoría en línea.

\section{ABSTRACT}

The article describes the role of teachers in virtual forms of teaching and learning in light of educational psychological models that have influenced distance education from its origins until today.

The type of educational psychological model, as well as the technological devices used, either afford or constrain the dimensions that constitute teaching practices in distance education. These two factors (affordance and constrains) must be considered in the design of teaching evaluation in online settings.

Keywords: evaluation of online teaching, computer-assisted instruction, teaching roles in virtual environments, online education, online tutoring. 
La enseñanza y el aprendizaje en las modalidades de educación a distancia que se apoyan en el uso de recursos tecnológicos, o que se desarrollan por completo en entornos virtuales, tienen características particulares, que a su vez configuran los roles que desempeñan tanto los profesores como los alumnos en diversas acciones educativas.

En la actualidad, los modelos de enseñanza en las modalidades a distancia se han diversificado ampliamente, por una parte influenciados por los desarrollos tecnológicos actuales que permiten hacer coincidir la participación de profesores y alumnos a través de sistemas de videoconferencias o de aulas virtuales, la utilización de chats o foros de discusión. Esta diversificación de modelos se debe, asimismo, a que en los programas formativos se contempla la creación de "comunidades de aprendizaje y de práctica” en las que se amplían las posibilidades de interacción de los usuarios con otras comunidades similares, de enseñanza formal e informal, centros de trabajo o investigación, e instituciones no sólo de enseñanza, sino de investigación, y en donde los encuentros y las posibilidades de aprender y enseñar se multiplican de forma considerable.

Como señalan Anderson y Dron (2011), las facilidades tecnológicas y las propuestas pedagógicas que han sustentado los modelos de educación a distancia han atravesado por tres generaciones en las que se han diversificado los recursos tecnológicos y los enfoques psicopedagógicos. Ninguna de estas generaciones, señalan los autores, ha proporcionado todas las respuestas a los complejos problemas que representa enseñar y aprender en esta modalidad.

En gran medida, las generaciones de modelos de educación a distancia se han desarrollado a partir de las tecnologías disponibles; a medida que se han desarrollado nuevos dispositivos tecnológicos, las posibilidades de comunicación e interacción han aumentado, y con ellas, los contenidos y aprendizajes que es posible transmitir y construir. Esto ha requerido que tanto profesores como alumnos, adquieran nuevas habilidades para manejar los dispositivos, y que se diseñen nuevas propuestas pedagógicas en las que se elija la "mezcla” más adecuada de tecnologías y enfoques psicopedagógicos.

Los autores señalan que la primera generación de modelos de educación a distancia se apoyaba en posturas psicopedagógicas cognitivo-conductuales y en tecnologías que posibilitaban la comunicación mediante medios impresos o audiovisuales, como la televisión y el radio. La comunicación entre los participantes en estos modelos, sólo podía realizarse de uno a uno, y la interacción con los recursos 
se realizaba mediante la lectura de textos, y la escucha o visión pasivas de programas de radio y/o televisión (ver tabla 1). El rol del alumno en esta primera generación era pasivo y su participación se limitaba a ver y escuchar. En la tabla 1 puede observarse cómo a partir de la modificación de los enfoques psicopedagógicos y del desarrollo de nuevas tecnologías, se ha ido dando paso a formas de interacción, no sólo de uno a uno, sino de uno a varios (participante-grupo), e incluso entre grupos (redes).

\begin{tabular}{|c|c|c|c|c|c|c|}
\hline $\begin{array}{c}\text { Fundamentos } \\
\text { Psico- } \\
\text { pedagógicos }\end{array}$ & Tecnologías & $\begin{array}{c}\text { Actividades } \\
\text { de } \\
\text { aprendizaje }\end{array}$ & $\begin{array}{c}\text { Unidad de } \\
\text { análisis de } \\
\text { aprendizaje } \\
\text { (granularidad) }\end{array}$ & $\begin{array}{c}\text { Unidad de } \\
\text { análisis de } \\
\text { contenido } \\
\text { (granularidad) }\end{array}$ & $\begin{array}{c}\text { Evaluación } \\
\text { basada en: }\end{array}$ & $\begin{array}{c}\text { Rol del } \\
\text { profesor }\end{array}$ \\
\hline $\begin{array}{c}\text { Cognitivo- } \\
\text { conductuales }\end{array}$ & $\begin{array}{c}\text { Medios } \\
\text { Imasivos: } \\
\text { Impresos, } \\
\text { TV, radio, } \\
\text { comunicación } \\
\text { uno a uno. }\end{array}$ & $\begin{array}{c}\text { Leer, ver y } \\
\text { escuchar. }\end{array}$ & Individual & $\begin{array}{c}\text { Fina: Basada } \\
\text { en scripts } \\
\text { (guiones) y } \\
\text { construida } \\
\text { desde "cero". }\end{array}$ & Recuerdo & $\begin{array}{c}\text { Creador/, } \\
\text { Diseñador de } \\
\text { contenidos: } \\
\text { "el sabio en } \\
\text { escena". }\end{array}$ \\
\hline $\begin{array}{c}\text { Conferencias } \\
\text { (audio, video, } \\
\text { y Web), } \\
\text { comunicación } \\
\text { individual y } \\
\text { colectiva. }\end{array}$ & $\begin{array}{c}\text { Discusión, } \\
\text { creación y } \\
\text { construcción } \\
\text { del } \\
\text { conocimiento. }\end{array}$ & Grupal & $\begin{array}{c}\text { Media: } \\
\text { apoyada en el } \\
\text { andamiaje y } \\
\text { guiada por el } \\
\text { profesor. }\end{array}$ & $\begin{array}{c}\text { Síntesis: } \\
\text { Ensayos }\end{array}$ & $\begin{array}{c}\text { Líder de la } \\
\text { discusión, } \\
\text { guía y } \\
\text { acompañante } \\
\text { en el proceso. }\end{array}$ \\
\hline
\end{tabular}

Tabla 1. Síntesis de Modelos Pedagógicos de Educación a Distancia. Adaptación de Anderson y Dron (2010)

Lo que interesa para esta discusión es principalmente, la forma, cómo ha cambiado el rol del profesor y cómo esto puede afectar la manera en que se aborda la evaluación de la docencia en nuevos entornos tecnológicos como la Web 2.0, las redes sociales y los sistemas de agregación de usuarios por recomendación de otros.

Como puede observarse en la tabla 1, el papel del profesor en la primera generación de modelos de educación a distancia era principalmente el de creador del contenido, aquel que sabía todo y definía todo; el profesor era el centro de la escena. En la segunda generación dominada por los enfoques constructivistas, el rol del profesor es el de líder, ya no es el centro del proceso de enseñanza-aprendizaje; su tarea es la de guiar al alumno, apoyarlo, estar a su lado y proporcionarle los andamios o ayudas necesarias para que vaya más allá del recuerdo y avance hacia la comprensión y síntesis de los contenidos. 
En la última generación de modelos, aún cuando los actores siguen siendo los mismos: el maestro, los estudiantes y el contenido, el desarrollo de relaciones entre estos tres se incrementa a partir del papel crítico que desempeña la interacción entre el estudiante y el contenido, el mismo que se individualiza a nivel personal y se materializa a través de la creación de artefactos. El estudiante no es más un consumidor de contenidos, sino su propio creador; el rol del profesor en esta nueva generación de modelos, de acuerdo con Anderson y Dron (2011), es el de un acompañante crítico, un compañero de viaje.

En consonancia con lo planteado líneas arriba, consideramos que los diferentes modelos son complementarios, ya que tienen diferentes finalidades y pueden usarse de manera individual o grupal, dependiendo de los contenidos a enseñar (conocimientos, procesos de pensamiento crítico, creatividad, etc.), y de los niveles de apropiación que se quieran impulsar en los participantes acerca de estos contenidos.

Los modelos conectivistas, que empiezan a dominar en la actualidad la escena educativa de la educación a distancia, están construidos sobre la base de un enfoque constructivista del aprendizaje donde el conocimiento se construye en un contexto que incluye no sólo redes y grupos externos a los participantes en un curso o programa educativo, sino considerandolas propias historiasypredilecciones delos participantes. Aún cuando el aprendizaje se apoya casi por completo en las interacciones sociales, las diversas partes involucradas pueden comunicar el conocimiento de forma más o menos efectiva, sean o no profesores o estudiantes. Incrementar el aprendizaje puede verse fortalecido por una comprensión de cómo aprenden las personas; en este contexto, los enfoques cognitivo conductuales, constructivistas y conectivistas, pueden constituir herramientas heurísticas útiles para seleccionar los enfoques psicopedagógicos más apropiados.

En línea con esta propuesta de utilizar de manera complementaria los enfoques psicopedagógicos al aprendizaje y la enseñanza, se encuentra la idea de que los instrumentos para evaluar la docencia en los diferentes modelos de educación a distancia deben considerar las formas que adoptan las interacciones entre los participantes y de éstos con el contenido, con las diversas figuras docentes, así como los resultados de aprendizaje que se espera lograr. Resulta, además, necesario considerar que el rol "del que enseña" se encuentra ampliamente distribuido entre los participantes, los dispositivos tecnológicos y el diseño mismo de los contenidos. Los tutoriales, por ejemplo, particularmente los tutoriales inteligentes, proporcionan ayudas que se encuentran ya construidas dentro del propio sistema tecnológico. 
De tal manera, que el rol del profesor está ya inserto en este entorno, por lo que la evaluación de la docencia podría realizarse mediante un análisis del tipo de ayudas que se han insertado en el dispositivo tecnológico. Éstas y otras consideraciones deberán ser tomadas en cuenta por quienes desarrollan propuestas para evaluar y formar docentes en ambientes virtuales. En la figura 1 se representa de forma gráfica un modelo conectivista de enseñanza-aprendizaje. Como puede observarse, los individuos se relacionan entre sí, no sólo al interior de sus instituciones, sino con otras externas. Las letras encerradas en círculos representan a los individuos, en donde la letra "a” representa a un alumno regular que interactúa con su maestro (M), y además, con otros alumnos de su mismo nivel (otras "a" minúsculas), y con otros alumnos de mayor experiencia y conocimiento que pueden haber ya cursado un programa particular de enseñanza, que se representan con la letra "A". Lo alumnos establecen también relaciones con sus tutores ( $\mathrm{T}$ ), con expertos (E) y sus colaboradores (C) en diversas instituciones externas como podrían ser empresas (Nacionales: $\mathrm{N}$, e Internacionales: I), otras universidades (Nacionales: N, e Internacionales: I) e instituciones tales como organizaciones civiles y organizaciones no gubernamentales (Nacionales: N, e Internacionales: I).

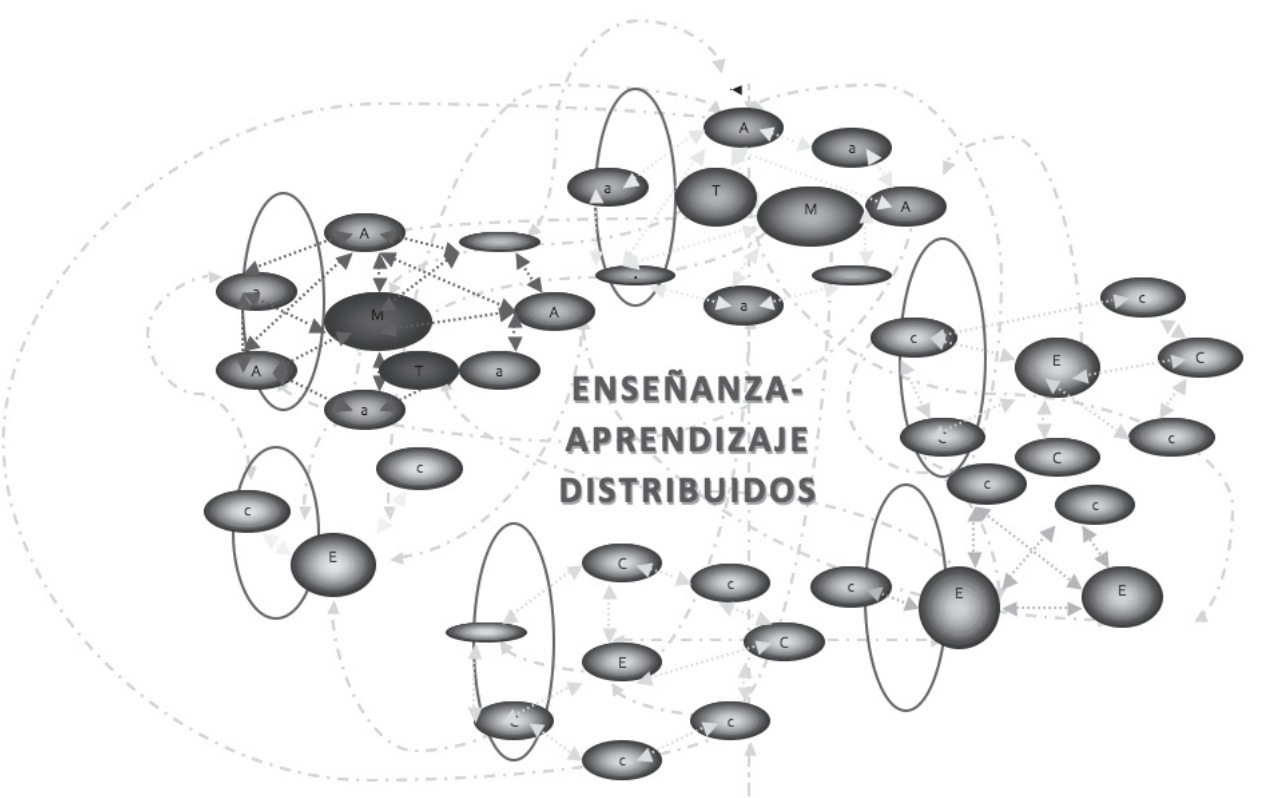

Figura 1. Se muestra una representación esquemática de un entorno conectivista de enseñanzaaprendizaje 
De esta manera, en un modelo conectivista, el conocimiento y el aprendizaje se encuentran distribuidos entre los nodos (individuos) y las redes que los interconectan. En este modelo la enseñanza se realiza a nivel horizontal, de forma colaborativa, por lo que resultan de particular importancia las interacciones discursivas entre pares (García-Cabrero y Pineda, 2010), así como la forma en que éstas impactan la evolución en la construcción de conocimientos (Guilar y Loring, 2008).

\section{LAS FUNCIONES DEL PROFESOR EN ENTORNOS VIRTUALES}

La modificación de los entornos de enseñanza-aprendizaje, como consecuencia del uso de las tecnologías de la información y la comunicación, implica, a su vez, un cambio en las formas en las que se lleva a cabo la enseñanza. McDonald y Reushle (2002) plantean que uno de los principales cambios o modificaciones ocurre en la filosofía de enseñanza que cada profesor tiene o ha desarrollado a lo largo de su trayectoria docente. Las formas de interacción con los alumnos, las expectativas tanto del profesor como de los alumnos, y los patrones de trabajo y actividades se configuran de manera diferente. Las labores y tareas que desempeña el profesor, por consecuencia, se diversifican y se complementan con la interiorización de diversos roles.

Cossetti (2000) señala que el conocimiento y las habilidades que posee el profesor para impartir cursos tradicionales resultan insuficientes cuando se trata de procesos de enseñanza-aprendizaje en modalidades virtuales.

Los roles que desempeña un profesor en línea han dado lugar a una multiplicidad de términos utilizados para designar su labor, lo cual ha dado lugar a confusiones respecto de las funciones que desempeñan los profesores en entornos virtuales.

$\mathrm{Al}$ respecto, Páez (2010) señala que las funciones que desempeña el profesor en los entornos virtuales de enseñanza-aprendizaje pueden ser variadas, por lo que resulta necesario delimitarlas lo más precisamente posible. De acuerdo con el autor, estas funciones pueden ser las de: facilitador, diseñador, mentor, evaluador, motivador, moderador, orientador y experto en contenido. El énfasis en cualquiera de estas funciones dependerá de las características del entorno virtual de enseñanza. Así, en modalidades a distancia donde los cursos se encuentran previamente diseñados, el rol principal del profesor consistirá en evaluar las tareas de los alumnos, moderar las interacciones, y resolver dudas sobre los contenidos disciplinares, dejando de lado su papel como diseñador. 
Los modelos actuales de educación a distancia muestran una fuerte tendencia a delimitar las funciones docentes asociadas a dos roles principales: en primer lugar, el de profesor-asesor; y en segundo lugar, el de profesor-tutor.

El profesor-asesor tiene como función principal promover el aprendizaje significativo de los alumnos, aunque también puede desempeñarse como diseñador de contenidos y de materiales didácticos que forman parte del entorno virtual de aprendizaje. Por su parte, el profesor-tutor, es aquel que ofrece una orientación sistemática al alumno para apoyar su avance académico conforme a sus necesidades $\mathrm{y}$ requerimientos particulares.

Ambas figuras docentes, deben contar con conocimientos psicopedagógicos sólidos, por lo que la capacidad para planificar es una de las competencias principales, que junto con otras, permiten que el profesor cumpla con la función docente en entornos virtuales de aprendizaje. Esto constituye lo que Ryan, Scott, Freeman y Patel (2000) denominan el rol pedagógico del profesor, y que Cabero (2004) considera como la función académica del docente en línea.

Las tareas que desempeña el profesor relacionadas con su rol pedagógico implican entre otras cosas, la creación del conocimiento especializado, centrar la discusión sobre los puntos críticos, contestar preguntas, responder a las diferentes contribuciones de los estudiantes y sintetizarlas.

Diversos autores (Adell y Sales, 2000; Berge, 1995; Cabero, 2004; Llorente, 2006; Valverde y Garrido, 2005) coinciden en que los roles del profesor no sólo se reducen a esta función, es decir, existen otras tareas que el profesor en línea tiene que desempeñar. $\mathrm{Al}$ respecto, Llorente (2006) considera que las funciones que debe realizar el profesor que se desempeña en entornos virtuales son: técnica, organizativa y social.

\section{Función técnica}

Los procesos de educación que se desarrollan en la virtualidad demandan que el profesor sea capaz de utilizar todos los recursos que ofrecen las tecnologías de la información y la comunicación. Es responsabilidad del docente utilizar de forma correcta las herramientas de comunicación que proporciona el entorno virtual. Con esto se garantiza, hasta cierto punto, que los aspectos técnicos no serán un obstáculo para que el proceso formativo se desarrolle de forma óptima. En este sentido, también es responsabilidad del profesor asegurarse de que los alumnos comprendan 
el funcionamiento técnico del entorno virtual de aprendizaje, y en caso de no ser así, proporcionar las ayudas y orientaciones necesarias para que el alumno participe sin mayores dificultades en las actividades programadas. Dentro de esta función se puede considerar también, la capacidad del docente para explotar todos los recursos que el entorno virtual proporciona para alcanzar las metas de aprendizaje y enseñanza.

Desempeñarse como profesor en línea supone también incorporar y/o modificar los materiales y recursos dentro del entorno virtual, dicha tarea implica capacidad para interactuar con el entorno de manera eficiente.

\section{Función organizativa}

Las actividades que se pueden consideran dentro de esta función se encuentran relacionadas con la capacidad que tiene el docente para explicar las normas de funcionamiento dentro del entorno; estructurar las formas de comunicación entre alumnos, y entre profesor y alumnos; ofrecer información de forma eficiente a los alumnos; organizar el trabajo en grupo; y facilitar la coordinación entre los participantes.

\section{Función social}

Berge (1995), Sánchez (2001), Barker (2002) y Cabero (2004), según cita Llorente (2006), señalan que la función social es una de las tareas que cobra mayor relevancia para facilitar que los estudiantes se desarrollen con éxito en las actividades y que se involucren en los programas de educación a distancia que se desarrollan en entornos virtuales. Es tarea del docente minimizar las situaciones de carácter afectivo que puedan interferir con el buen desempeño e integración del estudiante, tales como: aislamiento, pérdida, o falta de motivación. De acuerdo con Llorente (2006) existen determinadas actividades que el profesor puede llevar a cabo a fin de propiciar un clima que favorezca el desarrollo adecuado de las actividades de enseñanza aprendizaje, éstas son:

- Dar la bienvenida a los estudiantes que participan en el proceso formativo.

- Impulsar a los estudiantes a ampliar y desarrollar los argumentos presentados por sus compañeros, a fin de garantizar una mayor interacción entre los participantes.

- Integrar y conducir las intervenciones.

- Animar y estimular la participación. 
- Proponer actividades que faciliten que los participantes se conozcan entre sí.

- Dinamizar la acción formativa y el trabajo en red.

Por su parte Ugaz (s.f.) centra su análisis de las funciones docentes en entornos virtuales en el rol de tutoría. La autora señala, que aunque existen diversos modelos de tutoría, es posible determinar algunas funciones que son indiscutiblemente inherentes a esta labor. El tutor en la educación a distancia es responsable del seguimiento continuo, de los logros y dificultades encontradas por los estudiantes, es decir, acompaña al alumno durante su proceso de aprendizaje. Para ello, es necesario que el tutor cuente con una sólida formación académica y que tenga un manejo adecuado de las herramientas informáticas y del entorno virtual de enseñanza para que con ello pueda desempeñar tres tareas primordiales:

- Apoyar el proceso de auto-aprendizaje de los alumnos (Función Académica).

- Realizar funciones de enlace entre el alumno y la institución educativa (Función de Nexo).

- Orientar al estudiante y proporcionar apoyos para su formación integral (Función de orientación).

Cada una de las funciones que han sido descritas líneas arriba y representan aspectos imprescindibles para desempeñarse como docente en entornos virtuales de aprendizaje. Estos aspectos constituyen también elementos indispensables que deben ser incluidos en el desarrollo de procesos evaluativos de la calidad de la docencia en ambientes virtuales.

En relación con las funciones que lleva a cabo el profesor en entornos virtuales, Goold, Coldwell y Craig (2010) llevaron a cabo un estudio en la Universidad de Deakin (Australia) analizando cuatro módulos de aprendizaje que son cursados en modalidad virtual, es decir, sin ningún componente presencial. Para llevar a cabo el análisis consideraron las tres funciones docentes descritas líneas arriba considerando una función adicional, denominada "contenido" (Kim, Lee y Lim, 2010) y que hace referencia a todas aquellas actividades del profesor relacionadas directamente con los contenidos de aprendizaje y la didáctica de los mismos. Sorprendentemente los resultados encontrados indican que un alto porcentaje (78\%) de las intervenciones que realiza el profesor son de naturaleza organizativa, es decir, se encuentran orientadas a estructurar las formas de comunicación entre alumnos, organizar el trabajo en grupo, y facilitar la coordinación entre los participantes, entre otras más. En contraste, sólo el 18\% de las intervenciones que realiza el profesor se refieren a 
aspectos del contenido de aprendizaje, mientras que un $3 \%$ a aspectos relacionados con la función social, y un 1\% a aspectos técnicos.

Lo anterior permite inferir que en los entornos virtuales de enseñanza la función organizativa cobra especial relevancia, lo cual no implica que el resto de las funciones carezcan de importancia. A la luz de los resultados del estudio, conducido por Goold, Coldwell y Craig (2010), se plantea la necesidad de realizar este tipo de análisis en diversos contextos, y así, contar con un espectro de resultados más amplio que permita confirmar si lo encontrado por Goold, Coldwell y Craig (2010) se sostiene. Esta necesidad deberá impactar las formas que adopte la evaluación de la docencia, al igual que deberán reflejar los roles y el peso relativo de los mismos en la tarea de enseñar.

\section{EVALUACIÓN DEL ROL DEL DOCENTE EN AMBIENTES VIRTUALES: UNA PERSPECTIVA PSICOEDUCATIVA}

Para Fish y Wickersham (2009) una de las tareas primordiales del profesor en entornos virtuales consiste en promover el involucramiento activo de los estudiantes, comprometiéndolos a pensar de manera crítica, así como organizar y planificar la enseñanza, llevando a cabo un seguimiento y evaluación continuos del desempeño de los alumnos. Estas dimensiones de la acción del docente, al igual que las funciones descritas anteriormente, constituyen parámetros a partir de los cuales se pueden diseñar estrategias e instrumentos para la evaluación de la docencia en línea.

Uno de los modelos más aceptados en la actualidad, sobre el que se han basado diversas estrategias de análisis y evaluación de la práctica docente en entornos virtuales, es el caso de la Universidad de Athabasca, Canadá (The Community of Inquiry Model) desarrollado por Anderson, Rourke, Garrison y Archer (2001). Estos autores han planteado que el desempeño del profesor se manifiesta en los entornos virtuales a través de tres aspectos esenciales que definen su labor:

- Presencia docente: que implica realizar funciones de diseño instruccional, facilitación de la discusión y la colaboración, así como la instrucción directa a los alumnos.

- Presencia cognitiva: que incluye apoyar a los participantes para construir y conformar el significado de los contenidos educativos a través de la reflexión y el discurso. 
- Presencia social: es la habilidad de los participantes (profesor y alumnos) para proyectar características personales y aparecer ante los otros como personas reales.

Los aspectos anteriores han sido utilizados como indicadores del desempeño del profesor, al igual que se han valorado a través del andamiaje proporcionado a los alumnos, tipo y nivel de conocimientos alcanzado, así como del clima social que genera el docente (Lajoie, García-Cabrero, Berdugo, Márquez, Espíndola y Nakamura, 2006; García-Cabrero, Márquez, Bustos y Miranda, 2008).

\section{RETOS Y DESAFíos DE LA EVALUACIÓN DE LA DOCENCIA EN ENTORNOS VIRTUALES}

Como se desprende de la discusión anterior, se requiere invertir mayores esfuerzos en el desarrollo de metodologías sofisticadas para poder valorar cabalmente la contribución particular que realizan los profesores en modalidades de enseñanza a distancia en entornos virtuales, así como en el diseño de instrumentos ad-hoc que den cuenta del desempeño docente. Se requiere, como señala Páez (2010), instalar una cultura de la evaluación de los profesores que participan en modalidades de enseñanza-aprendizaje a distancia, considerando además de los cuestionarios de opinión de los estudiantes, el diseño de estrategias e instrumentos de auto-evaluación, co-evaluación y heteroevaluación.

Para hacer realidad una propuesta como la que se describe resulta necesario abordar el refinamiento de los modelos existentes de desempeño docente, y derivar nuevos indicadores de actuación de los profesores en modalidades de educación a distancia que caractericen su práctica cuando desempeñan diferentes roles: tutor, asesor, mentor, especialista, entre otros.

\section{CONSIDERACIONES FINALES}

Es preciso señalar que aunque la literatura sobre el tema ha logrado un consenso más o menos coherente en relación con las funciones de la docencia en entornos virtuales, difícilmente podría concretarse un perfil docente único que dé cuenta de manera exhaustiva de cada una de las tareas o labores que desempeña el profesor en los entornos virtuales.

Ello implica asumir que el proceso de construcción del perfil del docente en los entornos virtuales de enseñanza debe realizarse de manera flexible y dinámica. 
La naturaleza diversa y cambiante de los entornos virtuales de aprendizaje influye de manera directa sobre el tipo de habilidades y tareas que el profesor deberá desarrollar como parte de su labor docente en este tipo de entornos. Así mismo, el contexto institucional resulta fundamental para poder orientar la construcción de dicho perfil. No será lo mismo un curso en línea a nivel licenciatura ofertado por la Open University en Inglaterra, que un curso en línea a nivel bachillerato ofertado por la Secretaría de Educación del Distrito Federal en México. Cada institución tiene sus propios modelos educativos y formas de trabajo en educación a distancia, por lo que estos aspectos constituyen elementos que seguramente impactarán en la labor docente, y consecuentemente, en el diseño de instrumentos y estrategias de evaluación de la docencia.

Resulta muy complejo especular en el momento actual acerca de los cambios en los roles de los participantes, los alumnos y el contenido en nuevos entornos tecnológicos, ya que ahora se avecina la Web 3.0, la utilización de los dispositivos móviles y/o la realidad aumentada. Posiblemente estos nuevos dispositivos no acarrearán, como lo señalan Anderson y Dron (2011), cambios paradigmáticos como los que se observaron en las anteriores generaciones de modelos de educación a distancia, en vista de que la naturaleza y forma de comunicación, aunque es más refinada, no cambiará mucho con las tecnologías emergentes. Lo que sí se vislumbra es un cambio hacia una enseñanza basada en objetos, contextualizada, donde el logro alcanzado por las comunidades y redes de aprendizaje estará en gran medida determinado por su capacidad de localizar objetos apropiados, gente y actividades adecuadas, cuya capacidad discursiva dialógica (Ravenscroft, 2011) será aún más importante que las redes que se generen. En estas redes los mensajes no estarán dirigidos a entidades o personas conocidas, sino a una multitud compuesta por inteligencias múltiples que se comportará como una entidad individual intencional.

Estos escenarios representarán, sin duda, desafíos y retos de gran complejidad para los diseñadores de estrategias e instrumentos de evaluación de la docencia en entornos virtuales.

\section{REFERENCIAS BIBLIOGRÁFICAS}

Adell, J.; Sales, A. (2000). Enseñanza online: elementos para la definición del rol del profesor. En: Cabero, J.; Gisbert, M.; et al. (Coords). Las Nuevas tecnologías para la mejora educativa. Sevilla: Kronos.
Anderson, T.; Dron, J. (2010). Three generations of distance education pedagogy. The International Review of Research in Open and Distance Learning, North America, 12, nov. 
Anderson, T.; Rourke, L.; Garrison, D. R.; Archer, W. (2001). Assessing teaching presence in a computer conferencing context. Journal of Asynchronous Learning Networks, 5 (2), 1-17.

Barker, P. (2002). Skill sets for online teaching. ED-MEDIA 2002 World Conference on Educational Multimedia, Hypermedia and Telecomunications.

Berge, Z. (1995). Facilitating Computer Conferencing: Recommendations From the Field. Educational Technology, 35 (1), (22-30).

Cabero, J. (2004). La función tutorial en la teleformación. En: Martínez, F.; Prendes, M. P. (Coords). Nuevas Tecnologías y Educación. Madrid: Pearson Educación.

Cosetti, A. (2002). Tutoring: A different background for the same role. Ed-Media 2002, Denver, AACE, (355-356).

Fish, W. W.; Wickersham, L. E. (2009). Best practices for online instructors. Reminders. The Quarterly Review of Distance Education, 10 (3), (279-284).

García-Cabrero, B.; Pineda, V. (2010). La construcción de conocimiento en foros virtuales de discusión entre pares. Revista Mexicana de Investigación Educativa, 15 (44), (85-111).

García-Cabrero, B.; Márquez, L.; Bustos, A.; Miranda, G. (2008). Análisis de los patrones de interacción en una comunidad virtual de aprendizaje. Revista de Electrónica de Investigación Educativa, 10 (1).

Guilar, J. D.; Loring, A. (2008). Dialogue and Community in Online Learning: Lessons from Royal Roads University. Journal of Distance Education, 22 (3), (19-40).

Kim, Y. J.; Lee, S.; Lim, M. (2010). An analysis on tutor's roles for facilitating critical thinking in online discussion. En: Abas, Z.; et al.(Eds.). Proceedings of Global Learn Asia Pacific 2010. AACE.

Lajoie, S.; García-Cabrero, B.; Berdugo, G.; Márquez, L.; Espíndola, S.; Nakamura C. (2006). The Creation of Virtual and
Face-to-Face Learning Communities: An International Collaboration Experience. Journal of Educational Computing Research, 35 (2), (163-180).

Llorente, M. C. (2006). El tutor en E-learning: aspectos a tener en cuenta. Edutec. Revista Electrónica de Tecnología Educativa, 20, enero.

McDonald, J.; Reushle, S. (2002). Charting the role of the online teacher in higher education: winds of change. ASCILITE Conference 2002. Auckland, Nueva Zelanda.

Páez, R. O. (2010). Evaluación de las funciones docentes en entornos instructivos virtuales (EIV). Certezas, cuestionamientos y sinceramiento. Revista Iberoamericana de Evaluación Educativa, 3 (1e), (147-158).

Ravenscroft, A. (2011). Dialogue and connectivism: A new approach to understanding and promoting dialogue-rich networked learning. The International Review of Research in Open and Distance Learning, North America, 12, enero.

Ryan, S.; Scott, B.; Freeman, H.; Patel, D. (2000).The virtual university.The Internet and resource-based learning. Londres: Kogan Page.

Sánchez, F. (2001). Las tutorías y la construcción de ambientes de aprendizaje en la educación abierta. Revista Electrónica del Centro de Investigaciones y Servicios Educativos, 1,1 .

Ugaz, (s.f.). Claves para desempeñar el rol de tutor en un programa virtual. [en línea] Disponible en: http:// www.elearningamericalatina.com/ radiografias/rad 17.php (consulta 2011, 8 de enero).

Valverde, J.; Garrido, M. C. (2005). La función tutorial en entornos virtuales de aprendizaje: comunicación y comunidad. Revista Latinoamericana de Tecnología Educativa, 4 (1). 


\section{PERFIL ACADÉMICO Y PROFESIONAL DE LAS AUTORAS}

Benilde García Cabrero. Profesora Titular del Departamento de Psicología Educativa y Desarrollo de la División de Estudios de Posgrado de la Facultad de Psicología de la UNAM. Coordina la Sede "Tecnologías de la Información y la Comunicación Aplicadas a la Educación” en la Residencia en Psicología Escolar del Programa de Maestría de la Facultad de Psicología de la UNAM. Es consejera técnica del Instituto Nacional para la Evaluación de la Educación (INEE), y representante del Consejo Académico del Área de Ciencias Biológicas, Químicas y de la Salud ante el Consejo Asesor del Sistema Universidad Abierta y Educación a Distancia de la UNAM.

E-mail: benilde@unam.mx

Vania Jocelyn Pineda Ortega. Licenciada en Psicología por la Facultad de Psicología de la UNAM, obtuvo el grado de Maestría en Nuevas Tecnologías Aplicadas a la Educación otorgado por la Universidad Carlos III de Madrid, la Universidad Autónoma de Barcelona y la Universidad de Alicante. Actualmente se desempeña como asistente de investigación en la Facultad de Psicología de la UNAM y como tutora del Sistema de Bachillerato a Distancia del Ministerio de Educación de la Ciudad de México.

E-mail: vania.pineda@gmail.com

\section{DIRECCIÓN DE LAS AUTORAS:}

Av. Universidad 3004,

Col. Copilco-Universidad, CP. 04510, Del. Coyoacán, México, D.F.

Fechas de recepción del artículo: 06/07/11

Fecha de aceptación del artículo: 05/09/11

\section{Como citar este artículo:}

García Cabrero, B.; Pineda Ortega, V. J. (2011). Evaluar la Docencia en Línea: Retos y Complejidades. RIED. Revista Iberoamericana de Educación a Distancia, volumen 14, $\mathrm{n}^{0}$ 2, pp. 63-76. 